\title{
Length-scale competition in the damped sine-Gordon chain with spatiotemporal periodic driving
}

\author{
David Cai and A. R. Bishop \\ Theoretical Division and Center for Nonlinear Studies, Los Alamos National Laboratory, Los Alamos, New Mexico 87545 \\ Angel Sánchez \\ Theoretical Division and Center for Nonlinear Studies, Los Alamos National Laboratory, Los Alamos, New Mexico 87545 \\ and Escuela Politécnica Superior, Universidad Carlos III de Madrid, \\ Avenida del Mediterráneo 20, E-28913 Leganés, Madrid, Spain
}

(Received 4 December 1992)

\begin{abstract}
It is shown that there are two different regimes for the damped sine-Gordon chain driven by the spatiotemporal periodic force $\Gamma \sin \left(\omega t-k_{n} x\right)$ with a flat initial condition. For $\omega>k_{n}$, the system first bifurcates at a critical $\Gamma_{c}(n)$ to a translating two-breather excitation from a state locked to the driver. For $\omega<k_{n}$, the excitations of the system are the locked states with the phase velocity $\omega / k_{n}$ in all the regions of $\Gamma$ studied. In the first regime, the frequency of the breathers is controlled by $\omega$, and the velocity of the breathers, controlled by $k_{n}$, is shown to be the group velocity determined from the linear dispersion relation for the sine-Gordon equation. A linear stability analysis reveals that, in addition to two competing length scales, namely, the width of the breathers and the spatial period of the driving, there is one more length scale which plays an important role in controlling the dynamics of the system at small driving. In the second regime the length scale $k_{n}$ controls the excitation. The above picture is further corroborated by numerical nonlinear spectral analysis. An energy-balance estimate is also presented and shown to predict the critical value of $\Gamma$ in good agreement with the numerical simulations.
\end{abstract}

PACS number(s): 03.40.Kf, 61.72.Lk

\section{INTRODUCTION}

Recently it has been shown that length-scale competition is crucial for understanding dynamics of both one-dimensional nonlinear Schrödinger (NLS) and onedimensional sine-Gordon ( $\mathrm{SG}$ ) systems driven by a static, spatially periodic parametric potential [1-3]. The picture that has emerged is that the length-scale competition between the width of a breather and the spatial period of the external potential controls the particlelike coherence of the breather. The work of Refs. [1-3] has also established criteria for the validity of collective-coordinate descriptions for the excitations in the systems. In the work of Refs. [4,5] it has been demonstrated that a damped SG chain with additive dc driving also exhibits length-scale competition.

In this article we study the periodic SG system additively driven by a spatiotemporal periodic potential with a small damping strength. We anticipate that two lengths are intrinsic to this system and can be separately controlled, one being the width of a breather excited by the driving, and the other the spatial period of the driving field. The creation of a breather mode can be expected from the extensive work on the one-dimensional damped SG system additively driven by a temporally periodic external force [6-14]. This work has shown that the system exhibits transitions from oscillating spatially uniform states locked to the periodic driving force, to solitonic breather excitations, to more spatially irregular, less coherent states which are in a low-dimensional chaotic regime described in terms of a small number of interacting collective modes. The frequency of the coherent breather mode, excited at the first bifurcation with increasing driving strength, is locked to the frequency of the driving. This frequency-locking mechanism enables us to dynamically control the width of a breather in our system, i.e., by controlling the temporal frequency of the driving.

In light of the works mentioned above, one might expect that (i) our system will lock to the external driving; (ii) above a critical strength there will be additional breather-type excitations whose time scale is controlled by the driving frequency; and (iii) there will be a spatial length scale competition between the width of a breather and the spatial period of the driving. Our investigation supports this scenario and reveals an additional spatial length scale that plays a significant role in the competitions between length scales in the system, namely, the wavelength of the lowest spatial modulational mode.

Direct numerical simulation has provided much insight for the behavior of our system. Combined with linear stability analysis, energy-balance estimates $[8,15]$, and nonlinear spectral analysis [16-18], we have been able to achieve a broad understanding.

The organization of this article is as follows. In Sec. II we define the model and summarize the main numerical results. In Sec. III we investigate the linear stability of the locked periodic traveling wave train and show the origin of one of the spatially unstable modes. In Sec. IV, by a simple energy-balance argument, we provide a 
further understanding of the transition from the periodic traveling wave trains to a breather-mode excitation. In Sec. V we describe nonlinear-spectral-analysis results for our system.

\section{MODEL AND THE NUMERICAL RESULTS}

The governing equation of our system is

$$
\begin{aligned}
& U_{t t}-U_{x x}+\sin U=-\alpha U_{t}+\Gamma \sin \left(\omega t-k_{n} x\right), \\
& \exp [i U(x, t)]=\exp [i U(x+L, t)], \\
& U(x, t=0)=U_{t}(x, t=0)=0,
\end{aligned}
$$

where $L$ is the total length of the system, $\alpha$ is the damping coefficient, $\Gamma$ is the driving strength, and $k_{n}=$ $2 \pi n / L$. We chose $\omega=0.9$ and $\alpha=0.1$ and studied the cases for $1 \leq n \leq 7$ and $L=24$. With this choice of $\omega$, we are in the low-amplitude, near-NLS regime of SG. Occasionally we will compare the results for $L=24$ with those for $L=36$ to give a more complete picture.

The method we used to numerically integrate the equations is based on the Strauss-Vázquez scheme ([19]; see Sánchez, Vázquez, and Konotop [20] for details and references on its application to perturbed nonlinear KleinGordon problems). Figure 1 summarizes the main numerical observations for $L=24$. There is a major separation between two regimes, regime $\mathrm{I}, n \leq 3$, i.e. $k_{n}<\omega$; and regime II, $n>3$, i.e., $k_{n}>\omega$. For regime II, if we seek an ansatz of the form $U\left(\omega t-k_{n} x\right)$, Eq. (1) is reduced to a nonlinear oscillator with an antidamping coefficient, i.e.

$$
u_{\zeta \zeta}+\sin u=\alpha^{\prime} u_{\zeta}+\Gamma \sin (W \zeta)
$$

where $W=\sqrt{k_{n}^{2}-\omega^{2}}, \alpha^{\prime}=\alpha \omega / W, W \zeta=\omega t-k_{n} x-\pi$, $u=U-\pi$. A corollary of the linear stability analysis

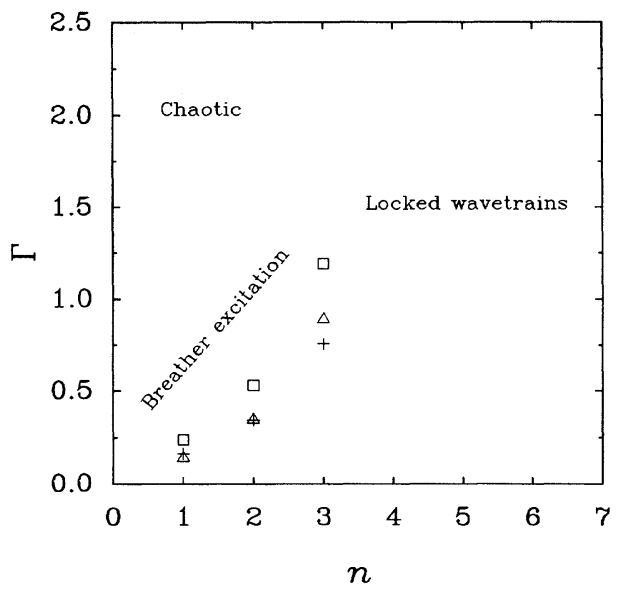

FIG. 1. Bifurcation diagram for the damped sine-Gordon chain driven by a spatiotemporal periodic force in the NLS regime. Parameters: $\omega=0.9, \alpha=0.1, L=24$. The crosses are the results obtained from the numerics, the squares from the linear stability analysis, and the triangles from the energybalance estimate. outlined below is that there is no linear stability for a wave train of the form $U_{0} \cos \left(\omega t-k_{n} x\right)$ in this regime II. Numerically we observed only stable periodic wave trains locked to the external driving, with the phase velocity $\omega / k_{n}$. These wave trains can be viewed as breather trains locked to the driving. This indicates that in this regime the $k_{n}$ mode controls the dynamics of the system and $k_{n}^{-1}$ is the dominant length scale.

Regime I exhibits far more complicated phenomena. In the following discussion we will confine ourselves to this regime unless otherwise specified. As is well known, the pure SG equation

$$
U_{t t}-U_{x x}+\sin U=0
$$

exhibits modulational instability, i.e., a periodic wave train of the functional form $U=U(\omega t-k x)$ is unstable (e.g. [21]). With the additional spatio-temporal periodic driving in Eq. (1), the system displays rather different behaviors. As for the case of the SG system driven by a temporally periodic force (see e.g., $[6,16]), n=0$ in our setting, for small $\Gamma$ below a certain critical value $\Gamma_{c}(n)$, our system for $n>0$ forms a periodic traveling wave locked to the driving from the flat initial background, i.e., of the form $U\left(\omega t-k_{n} x\right)$. With increasing $\Gamma$ the system exhibits a sharp transition at $\Gamma_{c}(n)$ to a new coherent state in which the breather excitation is observed and confirmed by the nonlinear spectral analysis. The frequency of the breathers is controlled by the temporal part of the external driving. Details of the nonlinear spectral analysis will be presented in Sec. V.

\section{LINEAR STABILITY ANALYSIS}

In the following, we present a linear stability analysis and show the origin of one of the spatially unstable modes. First, we seek a solution of the form $U=f\left(\omega t-k_{n} x+\theta\right)$ for the linearized version of Eq. (1) and find

$$
\begin{gathered}
f=U_{0} \cos \left(\omega t-k_{n} x-\theta\right), \\
U_{0}=\frac{\Gamma}{\sqrt{\left(k_{n}^{2}-\omega^{2}+1\right)^{2}+(\alpha \omega)^{2}}}, \\
\theta=-\arctan \left(\frac{k_{n}^{2}-\omega^{2}+1}{\alpha \omega}\right) .
\end{gathered}
$$

Now letting

$$
U=f+\eta,
$$

the linear stability is governed by

$\eta_{t t}-\eta_{x x}+\cos \left[U_{0} \cos \left(\omega t-k_{n} x-\theta\right)\right] \eta+\alpha \eta_{t}=0$.

Introducing $z=\omega t-k_{n} x-\theta$, we have from Eq. (4)

$-\eta_{x x}+2 k_{n} \eta_{x z}+\left(\omega^{2}-k_{n}^{2}\right) \eta_{z z}+\alpha \omega \eta_{z}+\cos \left[U_{0} \cos (z)\right] \eta=0$.

If we are concerned only with the instability in the amplitude of $f\left(\omega t-k_{n} x+\theta\right)$, i.e., the amplitude instability 
of the uniform wave train, we may assume $\eta(z)$ is independent of $x$. Hence the omission of $\eta_{x z}$. Alternatively, for large $L$, we may neglect the term $2 k_{n} \eta_{x z}$. Separating variables, we have

$$
\begin{aligned}
& \left(\omega^{2}-k_{n}^{2}\right) Z_{z z}+\omega \alpha Z_{z}+\left[K^{2}+\cos \left(U_{0} \cos z\right)\right] Z=0 \\
& X^{\prime \prime}+K^{2} X=0
\end{aligned}
$$

where $\eta=X Z$. For the amplitude instability of the uniform wave train, we have $K \equiv 0$ in Eq. (7) and we will retain the $k_{n}^{2}$ term without making the assumption that $L$ is large. Of course, in the large- $L$ approximation, the $k_{n}^{2}$ term in Eq. (6) should be omitted. Now letting

$$
\begin{aligned}
& Z=\exp \left[-\frac{\omega \alpha z}{2\left(\omega^{2}-k_{n}^{2}\right)}\right] Y, \\
& y=2 z+\pi
\end{aligned}
$$

and assuming small $U_{0}$, Eq. (6) becomes

$$
Y^{\prime \prime}+[\delta+\epsilon \cos y] Y=0 .
$$

This is the Mathieu equation with

$$
\begin{aligned}
\delta & =\frac{1}{\left(\omega^{2}-k_{n}^{2}\right)}\left(\frac{1}{4}-\frac{U_{0}^{2}}{16}-\frac{(\alpha \omega)^{2}}{16\left(\omega^{2}-k_{n}^{2}\right)}+\frac{K^{2}}{4}\right), \\
\epsilon & =\frac{U_{0}^{2}}{16\left(\omega^{2}-k_{n}^{2}\right)} .
\end{aligned}
$$

The parameter space $(\delta, \epsilon)$ of the Mathieu equation (9) is covered by bands of stable and unstable regions. Notice from Eq. (8) that $\eta$ is unbounded if $k_{n}>\omega$, since $Y(y)$ has either an asymptotically unbounded solution or a periodic one. It follows that there is no linear amplitude stability for regime II. Our system is near the critical point $\delta=1 / 4$ for small $\epsilon$ and low $K$. By increasing $U_{0}$, which is proportional to $\Gamma$, the parameters of Eq. (9) move across the boundary $\delta=1 / 4+\epsilon / 2+\cdots$ from the stable to the unstable region. We treat $\epsilon$ as the expansion parameter and define $\delta_{1}$ by

$$
\delta=1 / 4+\epsilon \delta_{1} .
$$

From the general theory of the Mathieu functions [22, 23 , we have the exponential factor

$$
\exp \left(\epsilon y \sqrt{1 / 4-\delta_{1}^{2}}\right)
$$

for $Y(y)$ in the asymptotic solution. Thus the boundary between the stable and the unstable regions for Eq. (6) is determined by

$$
2 \epsilon \sqrt{1 / 4-\delta_{1}^{2}}=\frac{\alpha \omega}{2\left(\omega^{2}-k_{n}^{2}\right)} .
$$

From Eq. (12) we have $\Gamma_{c}(1)=0.24, \Gamma_{c}(2)=0.53$, and $\Gamma_{c}(3)=1.19:$ see Fig. 1 and Table I. In determining $\Gamma_{c}$ we have set $K=0$, i.e., there is no spatial modulation on the amplitude. $\Gamma_{c}$ calculated in this way is the critical value for the onset of the amplitude instability of the uniform wave trains. We note in passing that
TABLE I. Comparison of the measured $\Gamma_{c}^{\text {measure }}$ with $\tilde{\Gamma}_{c}$ estimated by the energy-balance argument and $\Gamma_{c}$ estimated from linear stability analysis (see text).

\begin{tabular}{c|c|c|c}
\hline \hline & $n=1$ & $n=2$ & $n=3$ \\
\hline$\Gamma_{c}^{\text {measure }}$ & $0.1643-0.1645$ & $0.3421-0.3425$ & 0.7560 \\
\hline$\tilde{\Gamma}_{c}[$ from Eq. (16)] & 0.144 & 0.345 & 0.891 \\
\hline$\Gamma_{c}$ & 0.24 & 0.53 & 1.19 \\
\hline \hline
\end{tabular}

from the linear dispersion relation for the SG equation, $\omega_{k}=\sqrt{k^{2}+1}$, as $L$ gets larger, more and more discrete linear phonon modes shift below a fixed $\kappa$, where the driving is $\sin (\omega t-\kappa x)$. In our case there is only the $k=0$ phonon mode below $k_{1}$, two discrete linear phonon modes below $k_{2}$, and three below $k_{3}$; the dynamics as $L \rightarrow \infty$, such as the excitation bifurcation sequence, will be different from that depicted here.

Now we turn to the spatial instability of the uniform wave trains. At the boundary [Eq. (12)], $Z(z)$ has a solution periodic in space and time. Therefore the solution of Eq. (7) must be periodic in space with $K^{2}=(2 \pi m / L)^{2} \equiv$ $K_{m}^{2}, m=1, \ldots$. The solution $\cos \left(K_{m} x\right)$ is a modulation in space on the amplitude of $Z\left(\omega t-k_{n} x\right) . K_{1}$ is the lowest spatial modulational mode. The existence of the mode $K_{1}$ in the unstable region of the uniform wave train for $\Gamma \geq \Gamma_{c}$ signifies formation of a new state with a spatial structure whose length scale may be of order $K_{1}^{-1}$. $K_{1}^{-1}$ is yet another length scale in the system near $\Gamma_{c}$ in addition to the width of the excited breather, which is controlled by $\omega$, and the spatial period of the traveling background $\Gamma \cos \left(\omega t-k_{n} x\right)$ on which the breathers ride. The importance of this length-scale competition will be further illustrated with the nonlinear spectral analysis in Sec. V.

\section{ENERGY-BALANCE ESTIMATE}

In this section, we point out that a simple energybalance argument $[8,15]$ can predict the critical value of $\Gamma_{c}$ in good agreement with the numerical results. The simple ansatz we use is one breather with a static center, plus a sinusoidal traveling wave which is the solution for the linearized Eq. (1) [see Eq. (2)]:

$$
\begin{aligned}
U(x, t) & =U_{B}(x, t)+f \\
U_{B}(x, t) & =4 \arctan \left[\frac{\left(1-\Omega^{2}\right)^{1 / 2}}{\Omega} \frac{\cos (\Omega t-\Theta)}{\cosh \left[\left(1-\Omega^{2}\right)^{1 / 2} x\right]}\right]
\end{aligned}
$$

where $U_{B}(x, t)$ is the pure SG breather with $\Omega=\omega=0.9$. From the discussion above, the reason for this choice of $\Omega$ is clear; assuming the breathers to be traveling at a slow speed without much interaction, so that the "relativistic" correction to energy is small, our ansatz neglecting the interaction is reasonable. In Sec. V we will see that the above assumption is valid.

The change in energy in a period of time $T=2 \pi / \omega$ is

$\Delta H=\int_{-L / 2}^{L / 2} d x \int_{0}^{T} d t\left[\Gamma \sin \left(\omega t-k_{n} x\right)-\alpha U_{t}\right] U_{t}$ 
If we assume that the energy input to and output from the system in one period is balanced, i.e., $\Delta H=0$, and that $\tilde{\Gamma}_{c}$ is the smallest $\Gamma$ which can balance the above equation, then from Eq. (15) it is easy to obtain

$$
\begin{aligned}
\tilde{\Gamma}_{c}= & \frac{4 \alpha \sqrt{1-\omega^{2}}}{\omega} \frac{1}{I\left(k_{n}\right)} \\
& \times \arctan \frac{\sqrt{1-\omega^{2}}}{\omega \sqrt{1+\frac{1}{\omega^{2}\left(\sinh ^{-1}\left[L \sqrt{1-\omega^{2}} / 2\right]\right)^{2}}}},
\end{aligned}
$$

where

$$
\begin{array}{rl}
I(n)=\int_{-L / 2}^{L / 2} & d x \cos \left(k_{n} x\right) \\
\times & {\left[\sqrt{\cosh ^{2}\left(x \sqrt{1-\omega^{2}}\right)+\left(1-\omega^{2}\right) / \omega^{2}}\right.} \\
& \left.-\cosh \left(x \sqrt{1-\omega^{2}}\right)\right] .
\end{array}
$$

Comparing estimate (16) with our numerical simulations, we have the results in Table I (see also Fig. 1).

\section{NONLINEAR SPECTRAL ANALYSIS}

Numerical nonlinear spectral analysis is a powerful tool for diagnosing the solitonic content and radiation background for systems such as the weakly perturbed NLS and SG equations. The general theory and the numerical implementation in these cases are found in Refs. [16-18]. In the following, we will use this analysis to deepen our understanding of the behavior of our system and also to show that there is a simple mechanism which sets the excited breathers into translation.

As mentioned in Sec. II, our system forms a periodic traveling wave locked to driving from the flat initial background. With increasing $\Gamma$ the system exhibits a sharp transition at $\Gamma_{c}(n)$ to a new coherent state where, for $n=1,2$, a two-breather excitation is detected by the nonlinear spectral analysis. For $n=1$, these two breathers are out of phase and for $n=2$, they are in phase. Frequencies determined from the spectral analysis show that it is a good approximation that two breathers are locked to the temporal frequency $\omega$, see Fig. 2. For $n=2$, the frequencies of the breathers have a larger deviation from $\omega$. They oscillate between 0.75 and 0.9 . Also the two excited breathers are observed to run, i.e., translate along the system, at an average speed, 0.25 for $n=1,0.48$ for $n=2$, with some small fluctuations around these average speeds. Notice that the group velocity $V_{g}$ is

$$
V_{g}=\frac{k}{\sqrt{k^{2}+1}}
$$

from the linear dispersion relation for the $\mathrm{SG}$ equation

$$
\omega_{k}=\sqrt{k^{2}+1} .
$$

For $k_{n}=2 \pi n / L, V_{g}(n=1)=0.25, V_{g}(n=2)=0.46$, respectively. These values coincide with the average speed of the breathers within our er:or estimate. Since the value of $\omega$ is below the threshold of the phonon frequency
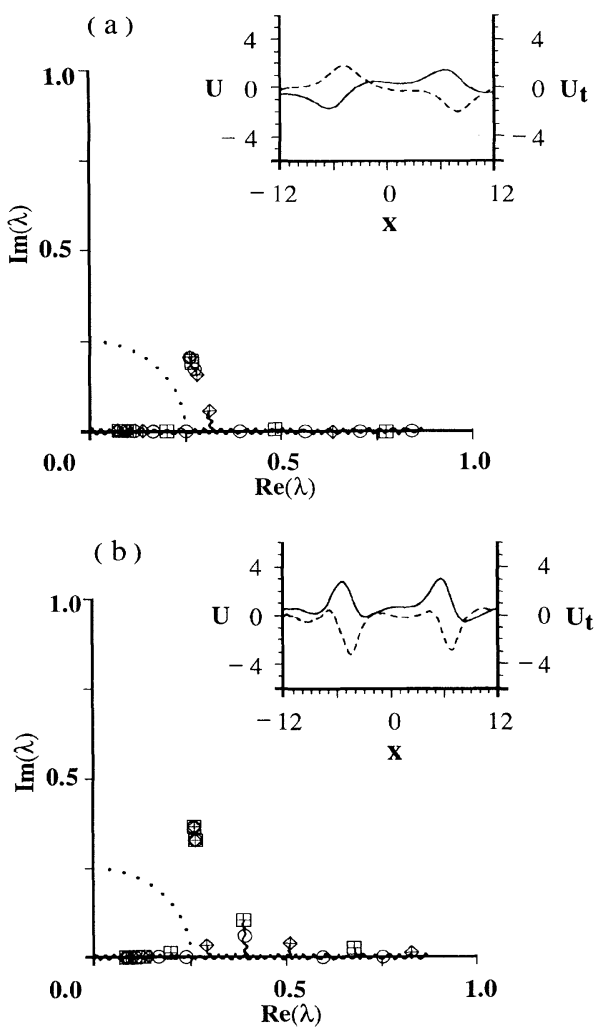

FIG. 2. (a) Field snapshots and the nonlinear spectral bands of the breathers at $t=4000$ for $n=1, \Gamma=$ 0.164534375 , and $L=24$. The solid line in the inset is $U(x, t)$, and the dashed line is $U_{t}(x, t)$. (b) The field snapshots and the associated nonlinear spectral bands of the breathers at $t=1988$ for $n=2, \Gamma=0.3425781$, and $L=24$.

[see Eq. (18)], only the spatial part of the driving can select the wave number of the phonons. The nonlinear spectrum indeed shows the $k_{n}$ excitation as a radiation spine attached to the real axis: it appears that these phonons drive the breathers into motion at the group velocity if the breathers are viewed as a disturbance around those $k_{n}$ 's. We have confirmed this assumption with $L=36$, see Table II. Our system has a driving force that couples the degrees of freedom in time and space. Nonetheless the above results indicate that in the parameter region of interest the frequency and the velocity of the excited breathers are controlled separately by the temporal and the spatial parts of the driving, respectively.

We also searched for a possible window of one-breather

TABLE II. $\quad V_{g}^{\text {measure }}$ is the measured average velocity of a breather. $V_{g}$ is the group velocity calculated from Eq. (17).

\begin{tabular}{c|l|l|l|l}
\hline \hline & $L=24$ & $L=24$ & $L=36$ & $L=36$ \\
\hline & $n=1$ & $n=2$ & $n=1$ & $n=2$ \\
\hline$V_{g}^{\text {measure }}$ & 0.25 & 0.48 & 0.18 & 0.34 \\
\hline$V_{g}$ & 0.25 & 0.46 & 0.17 & 0.33 \\
\hline \hline
\end{tabular}


excitation which might occur near $\Gamma_{c}$ following the traveling-wave region and before the onset of the twobreather excitation. We did not find any such window for $L=24$. We have examined $\Delta \Gamma / \Gamma_{c}$ to $10^{-3}$, where $\Delta \Gamma$ is the width of the window in which a one-breather excitation might exist.

As $\Gamma$ is increasing further above $\Gamma_{c}$, the centers of the two breathers start to move relative to each other, and it appears that they are weakly interacting. A third breather can be created if $\Gamma$ is sufficiently increased. The frequencies of these three breathers oscillate around $\omega$ and they exhibit rather complicated structure
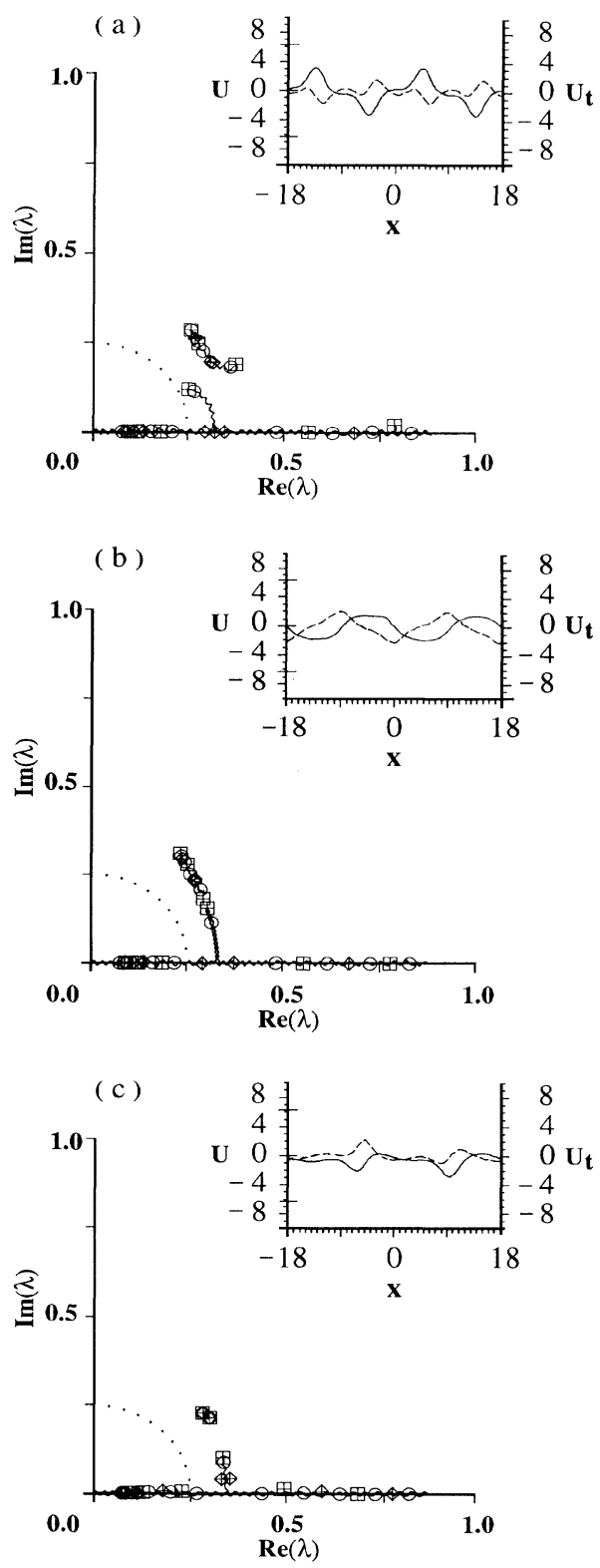

FIG. 3. Competition of excitations for $\Gamma=0.207, n=2$, and $L=36$ : (a) a four-breather excitation at $t=740$; (b) a locked state at $t=770$; (c) the final state at $t=1760$ after transients. in time. These two breathers have frequencies about 0.9 and 0.7 , respectively, and the other one has a very unstable structure often collapsing into anharmonic radiation and then reemerging. As a typical case, consider $L=24, n=1, \Gamma=0.3$ : for $0 \leq t<1900$, there are only two-breather excitations; for $t>1900$, there are three breathers. Many features of the system are still describable in terms of the behavior of these breathers. For example, the modulation of the total energy in time is discernable as the modulation due to the different frequencies of these breathers. These observations reinforce the notion that the collective-coordinate description for this nearly integrable system captures essential aspects of the system when a small number of degrees of freedom is involved.

For $n=3$, the transition is again sharp. The amplitude of the field is driven to the order of magnitude $2 \pi$. Then, new kink excitations together with breather excitations are detected by the nonlinear spectral analysis. The behavior of the loci of these solitonic spectral bands nonetheless indicates that the spatially coherent structure is less salient and is close to being destroyed: the field has the form of a traveling-wave packet with a large modulation than any spatially coherent excitation. It is hard to measure the group velocity of the wave packet in this case because it constantly alters its shape while traveling. However, a rough estimate of $V_{g}$ again agrees with the picture discussed above [see Eq. (17)].

To illustrate the competition of the three length scales and the importance of the $K_{1}$ mode in controlling the dynamics, we select the case of $L=36, n=2, \Gamma=$ 0.207 . From $t=0$ to $t \simeq 160$, the system is locked to the external driving as the sinusoidal amplitude of the field increases. Around $t>\sim 160$, four breathers are excited [see Fig. 3(a)]. Then a new state competes with this four-breather state. It has the form of a locked traveling wave with a large deviation away from sinusoid. Nonlinear spectral analysis shows a long radiation spine [see Fig. 3(b)]. The duration $\Delta t$ of each state is about 200-300 time units. These two states interleave with each other until $t \simeq 920$ after which the system settles into an attractor with two traveling breathers immersed in a lowamplitude radiation background. These two breathers have a pointlike spectral band in contrast to the relatively long spectral bands of the four breathers competing with the locked state [see Fig. 3(c)].

In all cases, $L=24$ and $36, n=1,2$, the system is attracted to a two-breather state at the first bifurcation after the transient if the length of the system can accommodate two such breathers whose frequencies are equal to $\omega$ in the lowest order of approximation. It appears that the amplitude instability with the $\cos \left(K_{1} x\right)$ modulation leads to the formation of the two-breather state. We therefore propose that it is the $K_{1}$ mode that is most important in controlling the dynamics in this parameter region.

\section{CONCLUSION}

We have identified two distinct regimes for our damped sine-Gordon chain driven by the spatiotemporal periodic 
potential. We have demonstrated that in regime I, of the three relevant length scales of our system, the $K_{1}$ mode appears to be the most important for controlling the low excitations, and that, in regime II, $k_{n}$ is the controlling length scale for the excitations of the system. We have discussed different selection mechanisms for determining the temporal frequencies of the breathers, controlled by the temporal part of the driving, and the running speed of the breathers, controlled by the spatial part of the driving. It appears that in our parameter region the presence of the traveling background causes the system to tend to form two running breather excitations at the first bifurcation in contrast to the case of pure temporal ac driving where the system is first attracted to a state of one nontranslating breather. We can expect, of course, that breathers excited by an ac-standing-wave-like driving will not run.

In summary, this work provides a further example supporting the concept of collective coordinates and competing length scales for soliton systems under relatively weak perturbation.

\section{ACKNOWLEDGMENTS}

We thank E.A. Overman for his nonlinear spectral code and his advice on the numerical inverse scattering transform, and R. Scharf and P. Lomdahl for discussions. We acknowledge support for this work by the U.S. DOE and partial financial support for A.S. by CICyT (Spain) under Project No. MAT90-0544.
[1] R. Scharf and A.R. Bishop, Phys. Rev. E 47, 1375 (1993).

[2] A. Sánchez, R. Scharf, A.R. Bishop, and L. Vázquez, Phys. Rev. A 45, 6031 (1992).

[3] R. Scharf, Yu S. Kivshar, A. Sánchez, and A.R. Bishop, Phys. Rev. A 45, R5369 (1992).

[4] J.C. Ariyasu and A.R. Bishop, Phys. Rev. B 35, 3207 (1987).

[5] J.C. Ariyasu and A.R. Bishop, Phys. Rev. A 39, 6409 (1989).

[6] A.R. Bishop, M.G. Forest, D.W. McLaughlin, and E.A. Overman, Physica D 23, 293 (1986).

[7] A. Mazor, A.R. Bishop, and D.W. McLaughlin, Phys. Lett. A 119, 273 (1986).

[8] A. Mazor and A.R. Bishop, Physica D 27, 269 (1987).

[9] A.R. Bishop, M.G. Forest, D.W. McLaughlin, and E.A. Overman, Phys. Lett. A 127, 335 (1988).

[10] A.R. Bishop, M.G. Forest, D.W. McLaughlin, and E.A. Overman, Phys. Lett. A 127, 335 (1988).

[11] A.R. Bishop, R. Flesch, M. G. Forest, D.W. McLaughlin, and E.A. Overman, SIAM J. Math. Anal. 21, 1511 (1990).

[12] G. Terrones, D.W. McLaughlin, E.A. Overman, and A.
Pearstein, SIAM J. Appl. Math. 50, 791 (1990).

[13] Niels Grønbech-Jensen, Boris A. Malomed, and Mogens R. Samuelsen, Phys. Rev. B 46, 294 (1992).

[14] Niels Grønbech-Jensen, Phys. Rev. B 45, 7315 (1992).

[15] P.S. Lomdahl and M.R. Samuelsen, Phys. Rev. A 34, 664 (1986).

[16] E.A. Overman, D.W. McLaughlin, and A.R. Bishop, Physica D 19, 1 (1986).

[17] M.G. Forest and D.W. McLaughlin, J. Math. Phys. 23(7), 1248 (1982).

[18] R. Flesch, M.G. Forest, and A. Sinha, Physica D 48, 169 (1991).

[19] W. Strauss and L. Vázquez, J. Comp. Phys. 28, 271 (1978).

[20] A. Sánchez, L. Vázquez, and V.V. Konotop, Phys. Rev. A 44, 1086 (1991).

[21] G.B. Whitham, Linear and Nonlinear Waves (Wiley, New York, 1974).

[22] J. Kevorkian and J.D. Cole, Perturbation Methods in Applied Mathematics (Springer-Verlag, New York, 1981).

[23] N.W. McLachlan, Theory and Application of Mathieu Functions (Oxford University, New York, 1947). 